\title{
A NOTE ON A SELECTION THEOREM
}

\author{
E. MICHAEL
}

ABstraCT. A recent selection theorem of O. N. Kolesnikov is generalized and its proof is simplified.

1. Introduction. The following result was recently obtained by $\mathrm{O}$. N. Kolesnikov [1, Theorem 1].

THEOREM $1.1[\mathbf{1}]$. Suppose $X$ is completely regular, $Y$ complete metric and locally pathwise connected, $C \subset X$ countable, and $\phi(x) \subset Y$ dense in $Y$ for all $x \in C$. Then there exists a continuous $f: X \rightarrow Y$ such that $f(x) \in \phi(x)$ for all $x \in C .1$

The purpose of this note is to give a proof of this theorem which is slightly simpler than the proof in $[\mathbf{1}]$, and which yields the following generalization.

THEOREM 1.2. Let $X, Y, C \subset X$ and $\phi(x) \subset Y$ be as in Theorem 1.1. Let $g: X \rightarrow Y$ be continuous with $g(X) \subset E$ for some closed, locally contractible $E \subset Y$, and let $A \subset X \backslash C$ be closed in $X$. Then there exists a continuous $f: X \rightarrow Y$ such that $f|A=g| A$ and $f(x) \in \phi(x)$ for $x \in C$. Moreover, given $\varepsilon>0$ one can choose $f$ so that $d(f, g)<\varepsilon$.

Theorem 1.1 follows immediately from Theorem 1.2, with $A=\varnothing$, by taking $g$ to be a constant map.

As Example 4.1 will show, the assumption in Theorem 1.2 that $g(X) \subset E$ for some closed, locally contractible $E \subset Y$ cannot be omitted. It is, however, not hard to show that it can be replaced by the assumption that $X$ is locally contractible at every $x \in C$.

\section{A lemma.}

LEMMA 2.1. Let $X$ be completely regular, $Y$ metric and locally pathwise connected, $A \subset X$ closed, $x^{*} \in X \backslash A$, and $D \subset Y$. Suppose also that $E \subset Y$ is closed and locally contractible and that $g: X \rightarrow E$ is continuous with $g\left(x^{*}\right) \in \bar{D}$. Then for each $\varepsilon>0$ there exists a closed, locally contractible $F \subset Y$ and a continuous $f: X \rightarrow F$ such that $f|A=g| A, d(f, g)<\varepsilon$ and $f\left(x^{*}\right) \in D$.

Proof. Let $W=\left\{y \in Y: d\left(y, g\left(x^{*}\right)\right)<\frac{1}{2} \varepsilon\right\}$. Let $V \subset W$ be a pathwise connected neighborhood of $g\left(x^{*}\right)$ in $Y$ such that $V \cap E$ is contractible over $W \cap E$, and pick $y^{*} \in V \cap D$. If $y^{*} \in E$, let $F=E$; if $y^{*} \notin E$, let $J$ be an $\operatorname{arc}^{2}$ in $V$

Received by the editors December 26, 1985.

1980 Mathematics Subject Classification (1985 Revision). Primary 54C65; Secondary 54D05.

Key words and phrases. Continuous selections, locally pathwise connected, locally contractible.

${ }^{1}$ This had previously been proved in [2] under stronger hypotheses on $X$ and $Y$.

${ }^{2}$ Recall that every pathwise connected Hausdorff space is arcwise connected (see, for example, [3, Corollary 31.6]). 
from $y^{*}$ to some $p \in E$ such that $J \cap E=\{p\}$, and let $F=E \cup J$. In either case, $F$ is locally contractible and $V \cap F$ is contractible over $W \cap F$. Pick a continuous $u:(V \cap F) \times \mathbf{I} \rightarrow W \cap F$ such that $u(y, 0)=y$ and $u(y, 1)=y^{*}$.

Let $U$ be open in $X$ with $x^{*} \in U \subset \bar{U} \subset g^{-1}(V) \backslash A$, and let $h: X \rightarrow \mathbf{I}$ be continuous such that $h\left(x^{*}\right)=1$ and $h(X \backslash U)=0$. Define $f: X \rightarrow F$ by $f(x)=g(x)$ if $x \notin U$ and $f(x)=u(g(x), h(x))$ if $x \in \bar{U}$. It is easy to check that this $f$ is well defined and satisfies our requirements.

3. Proof of Theorem 1.2. We assume that $C$ is infinite (the finite case is even simpler), and write $C=\left(x_{n}\right)$ with $x_{m} \neq x_{n}$ for $m \neq n$. Let $A_{0}=A$ and $A_{n}=$ $A \cup\left\{x_{1}, \ldots, x_{n}\right\}$ for $n \geq 1$. Apply Lemma 2.1 inductively to construct closed, locally contractible $E_{n} \subset Y$ and continuous $f_{n}: X \rightarrow E_{n}$ such that $E_{0}=E, f_{0}=g$, and, for all $n \geq 0, f_{n+1}\left|A_{n}=f_{n}\right| A_{n}, d\left(f_{n+1}, f_{n}\right)<2^{-n-1} \varepsilon$, and $f_{n+1}\left(x_{n+1}\right) \in \phi\left(x_{n+1}\right)$. The sequence $\left(f_{n}\right)$ is Cauchy, and thus converges uniformly to some $f: X \rightarrow Y$. This $f$ has the required properties.

REMARK. In both Lemma 2.1 and Theorem 1.2, the function $f$ is actually constructed to be $\varepsilon$-homotopic to $g$.

4. An example. I am grateful to Joseph Martin for kindly suggesting the following example, communicated to me by Mary Ellen Rudin.

EXAMPLE 4.1. A Peano space ${ }^{3} X$, a closed $A \subset X$, an $x^{*} \in X \backslash A$, and a dense, open $D \subset X$ such that, if $f: X \rightarrow X$ is continuous and $f(x)=x$ for $x \in A$, then $f\left(x^{*}\right) \notin D$.

PrOOF. Let $Z$ be a Peano space which fails to be locally contractible at exactly one point $p$, and let $X=Z \times \mathbf{I}$. Let $A=Z \times\{0\}$, let $x^{*}=(p, 1)$, and let $D=(Z \backslash\{p\}) \times \mathbf{I}$. It is not hard to check that this works.

\section{REFERENCES}

1. O. N. Kolesnikov, Sections on set-valued mappings, Vestnik Moskov. Univ. Ser. I Mat. Mekh. 1984, no. 2, 21-24 (= Moscow Univ. Math. Bull. 39 (1984), no. 2, 27-31).

2. E. Michael, Continuous selections and countable sets, Fund. Math. 111 (1981), 1-10.

3. S. Willard, General topology, Addison-Wesley, Reading, Mass., 1970.

Department of Mathematics, University of Washington, Seattle, WashingTON 98195

Current address (January-September 1987): Mathematisches Institut, Universität München, Theresienstrasse 39, D-8000 München, Federal Republic of Germany

${ }^{3}$ I.e., a connected, locally (pathwise) connected compact metric space. 